Bull. Austral. Math. Soc.

VOL. 52 (1995) [117-i36]

\title{
GROUPS 2-TRANSITIVE ON A SET OF THEIR SYLOW SUBGROUPS
}

\author{
Ben Brewster aNd Michael B. WaRd
}

\begin{abstract}
We classify, modulo the kernel of the action, finite groups $G$ that act 2-transitively on $S y l_{r}(G)$ for some prime $r$ dividing $|G|$. We furthermore prove that any finite group that acts 2-transitively on $S y l_{r}(G)$ for each prime $r$ is solvable and of nilpotent length at most 3 .
\end{abstract}

\section{INTRODUCTION}

By Sylow's Theorem, a finite group is always transitive when considered acting on $S y l_{p}(G)$, the set of Sylow p-subgroups of $G$, via conjugation. Here we consider the natural question of when that action is 2-transitive.

In Section 1 we clarify the notation and specify much of the background we need in the article. We then classify the finite groups $G$ which are 2-transitive and faithful on $S y l_{p}(G)$ for some prime $p$; Section 2 deals with solvable groups, while non-solvable groups are dealt with in Section 3. For Section 2, Huppert's classification of solvable 2-transitive permutation groups is fundamental. The classification of finite nonsolvable 2-transitive permutation groups is a consequence of the classification of finite simple groups and the work of Hering [9, 10] and Curtis, Kantor and Seitz [7]. In Section 3, we give a classification tailored to fit our needs and, in so doing, apply Hering's techniques to handle certain sporadic possibilites that do not seem to be covered elsewhere in the literature.

In perusing the resulting classification, we observe in Section 3 that any finite group $G$ that is 2-transitive (not necessarily faithful) on $S y l_{r}(G)$ for every prime $r$ dividing $|G|$ is solvable. We further examine the structure of such groups in Section 4, showing, for example, that any such group has nilpotent length at most 3 .

To stimulate thought, one might consider the following examples. The group $L_{2}\left(p^{n}\right)$ is 2-transitive on its Sylow $p$-subgroups. The symmetric group $S_{4}$ is 2 -transitive on each of its sets of Sylow subgroups as is the semidirect product $B$ of $S L_{2}(3)$ with its natural 2-dimensional module over $G F(3)$. Moreover, if $C$ is the semidirect product of a Sylow 7-subgroup of $S L_{3}(2)$ with its natural 3-dimensional module over $G F(2)$, then $C$ is 2-transitive on $S y l_{7}(C)$ and $B \times C$ is 2-transitive on each of its sets of Sylow subgroups.

Received 6th October, 1994

The second author thanks Utah State University for its hospitality during completion of this work.

Copyright Clearance Centre, Inc. Serial-fee code: 0004-9729/95 \$A2.00+0.00. 


\section{Notation and Preliminaries}

All groups $G$ considered here are finite.

Definition 1.1: Suppose $G$ acts on $\Omega$. $G$ is transitive on $\Omega$ provided whenever $\alpha, \beta \in \Omega$ there is $g \in G$ such that $\alpha^{g}=\beta$. $G$ is 2 -transitive on $\Omega$ provided whenever $\alpha_{1}, \alpha_{2} \in \Omega$ with $\alpha_{1} \neq \alpha_{2}$ and $\beta_{1}, \beta_{2} \in \Omega$ with $\beta_{1} \neq \beta_{2}$, there exists $g \in G$ such that for $i \in\{1,2\}, \alpha_{i}^{g}=\beta_{i}$

These are only slight perturbations of the standard definitions $([12, \mathrm{II}][\mathbf{2 3}])$. Usually they are applied to permutation groups (that is, faithful action) when $|\Omega|>1$.

If $G$ acts on $\Omega$ and $w \in \Omega$, we denote $G_{w}=\left\{g \in G \mid w^{g}=w\right\}$, the stabiliser of $w$ in $G$. Note that one convenient equivalence of 2 - transitivity is valid; namely, $G$ is 2-transitive on $\Omega$ if and only if $G$ is transitive on $\Omega$ and for some $w \in \Omega, G_{w}$ is transitive on $\Omega \backslash\{w\}$.

The following results are so central to the remainder of this article that we state them clearly here even though they are most likely to be well-known to the reader (see [23]).

Proposition 1.2. Let $G$ act faithfully and 2-transitively on $\Omega$ with $N \triangleleft G, N$ Abelian.

(i) $\quad N$ is a minimal normal subgroup of $G$ and $C_{G}(N)=N$.

(ii) $N$ is regular on $\Omega$, and for $w \in \Omega, G=N G_{w}$ with $N \cap G_{w}=1$.

(iii) For $w \in \Omega, G_{w}$ acting on $N \backslash\{1\}$ via conjugation is similar to $G_{w}$ acting on $\Omega \backslash\{w\}$.

Also, our definition that $G$ acting on $\Omega$ is similar to $\bar{G}$ acting on $\bar{\Omega}$ is that there exist a group isomorphism $\Phi: G \rightarrow \bar{G}$ and a bijection $\Psi: \Omega \rightarrow \bar{\Omega}$ such that for every $w \in \Omega$ and $g \in G,\left(w^{g}\right)^{\Psi}=\left(w^{\Psi}\right)^{g^{\Phi}}$.

Remembering Huppert's classification of 2-transitive solvable groups [11], naturally the group of semilinear transformations over a field plays a prominent role. We now establish our notation and mention some crucial properties of these groups.

For the prime $q$ and the positive integer $n$, let $\mathbb{K}$ denote the field of order $q^{n}$. Let $V=\{x \mapsto x+b \mid b \in \mathbb{K}\}, S=\{x \mapsto a x \mid a \in \mathbb{K}, a \neq 0\}$ and $A=\left\{x \mapsto x^{\alpha} \mid \alpha \in\right.$ $A u t(\mathbb{K})\}$. Note that $V$ is isomorphic to the additive group of $\mathbb{K}, S$ is isomorphic to the multiplicative group $\mathbb{K} \backslash\{0\}$ and $A$ is isomorphic the automorphism group of $\mathbb{K}$. Thus if $\bar{\sigma}$ is the Galois automorphism $a^{\bar{\sigma}}=a^{q}$ for any $a \in \mathbb{K},\langle\bar{\sigma}\rangle=A u t(\mathbb{K})$ and if $\sigma: x \mapsto x^{\bar{\sigma}},\langle\sigma\rangle=A$. We shall fix this meaning of $\sigma$ and use $\langle\sigma\rangle$ instead of $A$. We use $\Gamma\left(q^{n}\right)$ for the group of all semilinear transformations over $\mathbb{K}, \Gamma\left(q^{n}\right)=\left\{x \mapsto a x^{\alpha}+b \mid\right.$ $a, b \in \mathbb{K}, a \neq 0, \alpha \in A u t(\mathbb{K})\}$. The subgroups $V$ and $V S$ are normalised by $S$ and $\langle\sigma\rangle, V \cap S=1=V S \cap\langle\sigma\rangle, \Gamma\left(q^{n}\right)=V S\langle\sigma\rangle,|V|=q^{n},|S|=q^{n}-1,|\langle\sigma\rangle|=n$, and $S\langle\sigma\rangle$ is supersolvable. 
As permutations of $\mathbb{K}, V$ is a regular normal subgroup of $\Gamma\left(q^{n}\right)$ and $\Gamma\left(q^{n}\right)_{0}=$ $S\langle\sigma\rangle$. Note that if $S \leqslant U \leqslant \Gamma\left(q^{n}\right), V U$ is 2-transitive on $\mathbb{K}$. The article [17] specifies exactly which subgroups of $\Gamma\left(q^{n}\right)$ are 2-transitive on $\mathbb{K}$, but on the Sylow subgroups there are alternative approaches that we choose.

The action of $\langle\sigma\rangle$ on $S$ and $V$ via conjugation may be identified with the action of $\langle\bar{\sigma}\rangle$ on $\mathbb{K} \backslash\{0\}$ and $\mathbb{K}$ respectively. We shall make use of this identification. In particular, we shall think of $C_{S}\left\langle\sigma^{i}\right\rangle$ and $C_{V}\left(\sigma^{i}\right)$ as subsets of the fixed field of $\left\langle\vec{\sigma}^{i}\right\rangle$.

Using this notation we establish the following lemma.

Lemma 1.3. Let $\bar{G} \leqslant \Gamma\left(q^{n}\right)$ and let $\rho: \Gamma\left(q^{n}\right) \rightarrow\langle\sigma\rangle$ denote the natural mapping with kernel VS. Suppose that $\rho(\bar{G})=D \leqslant\langle\sigma\rangle$ with $D \neq 1$. Suppose that $U \leqslant S$ such that $U$ is not contained in any proper subfield of $\mathbb{K}$ which contains the fixed field of $D$.

Then $C_{\bar{G}}(U) \leqslant S$.

Proof: Let $x \in C_{\bar{G}}(U), x=v s d$ where $v \in V, s \in S$ and $d \in D$. For any $u \in U, u=u^{v s d}$ and so $\left(u^{d^{-1}}\right)^{s^{-1}}=u^{v}$. But $d \in N_{\bar{G}}(S)$ and $S$ is cyclic, and so $\left(u^{d^{-1}}\right)^{s^{-1}}=u^{d^{-1}}$.

Thus for any $u \in U, u^{d^{-1}}=u^{v}$. Hence $u^{-1} u^{d^{-1}}=u^{-1} u^{v}$, and so $[u, v] \in S \cap V=$ 1. By hypothesis $U \neq 1$, since otherwise $U$ is contained in the prime subfield of $\mathbb{K}$. Then $v$ is fixed by $U$ and so in $\bar{G}, v=1$.

It then follows that each element of $U$ is fixed by $d$. However, $U$ is not contained in any proper subfield containing the fixed field of $D$ and so cannot be fixed by $\langle d\rangle$, if $d \neq 1$. Thus $d=1$ and $x \in S$.

The following important result by Huppert [11] completely determines solvable 2-transitive permutation groups.

Proposition 1.4. Suppose $G$ is a solvable group which acts faithfully and 2-transitively on the set $\Omega$. Then, with 13 exceptions, there is a subgroup $\bar{G} \leqslant \Gamma\left(q^{n}\right)$ such that $G$ acting on $\Omega$ is similar to $\bar{G}$ acting on $\mathbb{K}=G F\left(q^{n}\right)$.

The 13 exceptions are explicitly represented in [11] and we shall use these representations to complete our classification of groups which are 2-transitive on Sylow subgroups.

We shall consistently use the bar notation for images of subgroups of $G$ in $\Gamma\left(q^{n}\right)$.

A little number theory comes into play. An often used result dating back to Zsigmondy helps us. We give reference to Huppert-Blackburn [13, IX, Theorem 8.3, p.508].

Proposition 1.5. (Zsigmondy). Suppose $q$ is prime and $n>1$ is an integer. Then there is a prime $r$ such that $r \mid p^{n}-1$ but $r \nmid p^{i}-1$ for $0<i<n$, and $r \nmid n$, 
unless $q$ is a Mersenne prime and $n=2$, or $q=2$ and $n=6$.

The prime $r$ will be called a Zsigmondy prime for the prime $q$ and integer $n$.

Finally, we shall be considering a group $G$ acting on its set of Sylow p-subgroups, $S y l_{p}(G)$, via conjugation. For $P \in S y l_{p}(G)$, its stabiliser in this action is $N_{G}(P)$ and we let $K_{p}=\bigcap_{x \in G} N_{G}\left(P^{x}\right)=$ core $N_{G}(P)$ denote the kernel of the action. Hopefully any other notation will be standard, see [12] and [8].

\section{Solvable groups $G$ 2-TRANSITIVE on $S y l_{p}(G)$}

In this section we shall classify those solvable groups which are faithful and 2transitive on $S y l_{p}(G)$ for some prime $p$.

Proposition 2.1. Suppose $G$ acts faithfully and 2-transitively on $S_{y} l_{p}(G)$ and suppose that this action is similar to a subgroup $\bar{G}$ of $\Gamma\left(q^{n}\right)$ acting on $\mathbb{K}=G F\left(q^{n}\right)$. If $\bar{P} \in S y l_{p}(G)$ and $\bar{P} \not V S$, then $p=2, q$ is a Mersenne prime and $n=2$.

Proof: Since $V S \triangleleft \Gamma\left(q^{n}\right)$, we are then supposing that no Sylow $p$-subgroup of $\bar{G}$ is contained in $V S$. Let $\bar{P} \in S y l_{p}(\bar{G})$.

Suppose there is a Zsigmondy prime $r$ for $q$ and $n$. Since $\left|\Gamma\left(q^{n}\right)\right|=q^{n}\left(q^{n}-1\right) n$ and because $\bar{G}$ is 2-transitive on $\mathbb{K}$, a Sylow $r$-subgroup of $\bar{G}$ is a Sylow $r$-subgroup of $\Gamma\left(q^{n}\right)$. Also, since $\left|\bar{G}: N_{\bar{G}}(\bar{P})\right|=\left|S y l_{p}(G)\right|=|\mathbb{K}|=q^{n}, N_{\bar{G}}(P)$ contains a Sylow $r$-subgroup of $\bar{G}$. Consequently, by choosing an appropriate conjugate of $\bar{P}$, we may suppose $N_{\bar{G}}(\bar{P})$ contains $\bar{R}$ where $\bar{R} \in S y l_{r}(S)$. Note $V$ is characteristic in $V S$ and since $S$ is cyclic, $V \bar{R}$ is characteristic in $V S \triangleleft \Gamma\left(q^{n}\right)$. Hence $V \bar{R} \triangleleft \Gamma\left(q^{n}\right)$.

Then $[\bar{R}, \bar{P}] \leqslant V \bar{R} \cap \bar{P}=1$ since $p \neq r$ and $p \neq q$. Hence $\bar{P} \leqslant C_{\Gamma\left(q^{n}\right)}(\bar{R})$. However, $\bar{R}$ is contained in no proper subfield of $\mathbb{K}$. Also, that $\bar{P} \not V S$ implies that if $\rho: \Gamma\left(q^{n}\right) \rightarrow\langle\sigma\rangle$ is the natural homomorphism with ker $\rho=V S$, then $\rho(\bar{G}) \neq 1$. Consequently by Lemma $1.3, C_{\bar{G}}(R) \leqslant S$. This gives the contradiction that $\bar{P} \leqslant V S$. Hence, there is no Zsigmondy prime for $q$ and $n$.

Suppose next that $q=2$ and $n=6$. Then $|S|=2^{6}-1=9 \cdot 7$ and $p=2$ or $p=3$. From Proposition 1.2, it is straightforward to deduce that $V \leqslant \bar{G}$. Since $V$ is a 2-group and $V \triangleleft G, p \neq 2$. If $p=3$, then, with $\rho$ as above, $\rho(G)=\left\langle\sigma^{2}\right\rangle$. The fixed field of $\left\langle\bar{\sigma}^{2}\right\rangle$ is $G F\left(2^{2}\right)$ and so if $\bar{R} \in S y l_{7}(G), \bar{R}$ is contained in no proper subfield of $\mathbb{K}$ which contains $G F\left(2^{2}\right)$ and so $C_{\bar{G}}(\bar{R}) \leqslant S$ by Lemma 1.3. But, as above, $[\bar{P}, \bar{R}]=1$ and so $\bar{P} \leqslant V S$, a contradiction. Thus it is impossible that $q=2$ and $n=6$.

From Proposition 1.5, it only remains that $p=2=n$ and $q$ is a Mersenne prime.

We note the converse and a bit of structure.

Proposition 2.2. Let $q$ be a prime and $n$ a positive integer.

(i) If $P \in S y l_{p}\left(\Gamma\left(q^{n}\right)\right)$ and $P \leqslant S$, then $\Gamma\left(q^{n}\right)$ is 2-transitive on $S y l_{p}\left(\Gamma\left(q^{n}\right)\right)$ 
and $V S / V$ is nilpotent.

(ii) If $p=2, q$ is a Mersenne prime and $n=2$, then if $U \leqslant \Gamma\left(q^{2}\right)$ such that $\left[\Gamma\left(q^{2}\right): U\right] \mid 2, U$ is 2- transitive on $S y l_{2}(U)$ and $\Gamma\left(q^{2}\right) / V$ is nilpotent.

The proof will be omitted since the comments in Section 1.1, Proposition 1.2 and the ideas from the proof of Proposition 2.1 lead to the proof. Moreover, in our article [3] most details are explicit.

Next we check the exceptions mentioned in Huppert's Theorem.

Proposition 2.3. If $G$ is faithful and 2-transitive on $S y l_{p}(G)$ but $G$ on $S y l_{p}(G)$ is not similar to a subgroup of $\Gamma\left(q^{n}\right)$ on $\mathbb{K}$, then $G=V G_{0}$ where one of the following holds:

(i) $|V|=3^{2}, G_{0} \cong S L(2,3), p=2$

(ii) $|V|=5^{2}, G_{0} \cong S L(2,3), p=2$

(iii) $|V|=5^{2}, G_{0} \cong S L(2,3) \star C_{4}, p=2$ (where $\star$ denotes central product)

(iv) $|V|=11^{2}, G_{0} \cong S L(2,3) \times C_{5}, p=2$

(v) $|V|=11^{2}, G_{0} \cong S L(2,3) \times C_{5}, p=5$

(vi) $|V|=23^{2}, G_{0} \cong G L(2,3) \times C_{11}, p=11$

(vii) $|V|=3^{4}, G_{0} \cong\left(D_{8} \star Q_{8}\right) C_{5}, p=2$.

Proof: The proof follows by calculating with the exceptions listed by Huppert [11] to check if point stabilisers contain a Sylow subgroup of $G$ as a normal subgroup.

\section{Nonsolvable groups $G$ 2-TRansitive on $S y l_{p}(G)$}

The classification of finite nonsolvable 2-transitive permutation groups is a consequence of the classification of finite simple groups. Interesting discussions are found in $[14,5,1]$. We include a sketch of the argument.

Suppose $G$ is a nonsolvable 2-transitive permutation group and let $M$ be a minimal normal subgroup of $G$. Either $M$ is regular or it is primitive and isomorphic to a nonabelian simple group.

If $M$ is primitive and simple, then $C_{G}(M)=1$ and so $G$ embeds in $\operatorname{Aut}(M)$. We therefore assume in this case that $M \leqslant G \leqslant A u t(M)$. Invoking the classification of finite simple groups, we must consider the cases where $M$ is alternating, of Lie type or sporadic.

When $M$ is alternating, the classical reference is Maillet [15]. When $M$ is of Lie type, Curtis, Kantor and Seitz [7] give a complete list of possibilities. Finally, when $M$ is sporadic, W. Kantor said in 1982 that the possibilities are "folklore" [14]. Nowadays, one may utilise the ATLAS [6]. For assume $M$ has a 2-transitive permutation representation. Then each point stabiliser is a maximal subgroup of $M$ and the corresponding permutation character is $1+\chi$ where $\chi$ is an irreducible character of $M$. For all but 
the largest sporadic groups, one can locate explicitly when that situation occurs in the ATLAS. The possibilities are given in Proposition 3.1 below. For the largest sporadic groups, where information on maximal subgroups is incomplete, note that the values of $1+\chi$ are the numbers of fixed points of elements of $M$ and, hence, must be nonnegative integers. It only takes a few minutes of looking at the character tables in the ATLAS to find there is no such $\chi$ for the large sporadics.

The ATLAS may also be used to check for the prospect that $G$ is 2- transitive, but $M$ isn't. That could only happen, of course, if $M<A u t(M)$, which is true for only 12 of the sporadic groups and, in each case, $[A u t(M): M]=2[6$, p.viii]. Thus, a $G$ of the sort we seek would have to equal $A u t(M)$. Let $n$ be the degree of the 2- transitive representation of $G$. The analysis as above could be used, but we'll give another simple-minded way to show that $M$ must be 2-transitive. If $M$ is not 2-transitive, then a point stabiliser $M_{\alpha}$ is not transitive on the points different from $\alpha$. In other words, $M_{\alpha} G_{\alpha \beta} \neq G_{\alpha}, \alpha \neq \beta$. Since $\left[G_{\alpha}: M_{\alpha}\right]=[G: M]=2$, we must have $G_{\alpha \beta} \leqslant M_{\alpha}, \alpha \neq \beta$. That gives $n-1=\left[G_{\alpha}: G_{\alpha \beta}\right]=\left[G_{\alpha}: M_{\alpha}\right]\left[M_{\alpha}: G_{\alpha \beta}\right]=2\left[M_{\alpha}\right.$ : $\left.G_{\alpha \beta}\right]$. Hence, $n$ is odd and $(n-1) / 2$ divides $\left|M_{\alpha}\right|$. By the primitivity of $M$, we know $M_{\alpha}$ is a maximal subgroup of index $n$ in $M$. For all but the Fischer group $F i_{24}^{\prime}$, the ATLAS shows that there is no maximal subgroup satisfying our arithmetic conditions. The character table for $\operatorname{Aut}\left(F i_{24}^{\prime}\right)$ shows there is no irreducible character $\chi$ with $1+\chi$ having nonnegative integer values. As before, that means $A u t\left(F i_{24}^{\prime}\right)$ has no 2-transitive representation. Therefore, in the sporadic case, $M$ itself must be 2 -transitive whenever $G$ is 2-transitive.

The proposition below summarises the situation. When convenient, a hint is given on the source of the permutation representation for the information of the reader. In the cases of isomorphism between simple groups, the groups may be listed more than once for the sake of completeness. Geometric phrases like "on points" refer to the projective space naturally corresponding to the group. We use ATLAS-like notation for groups and the structure of a point stabiliser.

Proposition 3.1. Suppose $G$ has a 2-transitive permutation representation and $M \leqslant G \leqslant A u t(M)$ where $M$ is a nonabelian simple group. Then we have one of the cases given in Table 1.

Proof: $[15,7]$ and the above discussion for the sporadic finite simple groups. $]$

If $M$ is regular then it is elementary Abelian, and our Proposition 1.2 holds. We may think of $G$ as acting on a vector space $V$ of dimension $n$ over $G F(p)$ with $M$ the group of translations and $G_{0}$ a group of linear transformations acting transitively on the nonzero vectors. Hering $\left[9\right.$, p. 443-444] gives examples for $G_{0}$ and in $[9,10]$ shows those are the only possibilities when there is a simple nonabelian composition factor that is alternating or of Lie type. 
TABLE 1.

\begin{tabular}{|c|c|c|c|c|}
\hline Case & $\mathrm{M}$ & degree & $M_{a}$ & representation \\
\hline ALT1 & $A_{n}, n \geq 5$ & $n$ & $A_{n-1}$ & $\begin{array}{l}\text { natural, extra one } \\
\text { when } n=6\end{array}$ \\
\hline ALT2 & $A_{5}$ & 6 & $N_{M}(P), P \in S y l_{5}(M)$ & $A_{5} \cong L_{2}(5)$ on points \\
\hline ALT3 & $A_{6}$ & 10 & $N_{M}(P), P \in S y l_{3}(M)$ & $A_{6} \cong L_{2}(9)$ on points \\
\hline ALT4 & $A_{8}$ & 15 & $2^{3}: L_{3}(3)$ & $\begin{array}{c}A_{8} \cong L_{4}(2) \text { on points } \\
\text { or hyperplanes }\end{array}$ \\
\hline ALT5 & $A_{7}$ & 15 & $L_{3}(2)$ & $\begin{array}{c}A_{7} \leq A_{8} \cong L_{4}(2) \text { on } \\
\text { points or hyperplanes }\end{array}$ \\
\hline LT1 & $L_{m}(q), m \geq 3$ & $\frac{q^{m}-1}{q-1}$ & $q^{m-1}: \frac{q-1}{(m, q-1)} \cdot P G L_{m-1}(q)$ & on points or hyperplanes \\
\hline $\mathrm{LT} 2$ & $L_{2}(q), q>3$ & $q+1$ & $\begin{array}{c}N_{M}(P), P \in S y l_{p}(M) \\
q=p^{m}\end{array}$ & on points \\
\hline LT3 & $U_{3}(q), q>2$ & $q^{3}+1$ & $\begin{array}{c}N_{M}(P), P \in S y l_{p}(M) \\
q=p^{m}\end{array}$ & on isotropic points \\
\hline LT4 & ${ }^{2} B_{2}(q), q>2$ & $q^{2}+1$ & $\begin{array}{c}N_{M}(P), P \in S y l_{2}(M) \\
q=2^{2 m+1}\end{array}$ & \\
\hline LT5 & ${ }^{2} G_{2}(q), q>3$ & $q^{3}+1$ & $\begin{array}{c}N_{M}(P), P \in S y l_{3}(M) \\
q=3^{2 m+}\end{array}$ & \\
\hline LT6 & $L_{2}(5)$ & 5 & $A_{4}$ & $L_{2}(5) \cong A_{5}$ \\
\hline LT7 & $L_{2}(9)$ & 6 & $A_{5}$ & $L_{2}(9) \cong A_{6}$ \\
\hline LT8 & $L_{4}(2)$ & 8 & $A_{7}$ & $L_{4}(2) \cong A_{8}$ \\
\hline LT9 & $L_{2}(8)$ & 28 & $\begin{array}{l}G_{\alpha}=N_{G}(P) \\
P \in S y l_{3}(G)\end{array}$ & $\begin{array}{l}P \Gamma L(2,8) \cong{ }^{2} G_{2}(3) \\
M \text { is not } 2 \text {-transitive }\end{array}$ \\
\hline LT10 & $C_{m}(2)$ & $\begin{array}{l}\left(2^{m-1}\right) \\
\left.2^{m} \pm 1\right)\end{array}$ & $G O_{2 m}^{ \pm}(2)$ & $\begin{array}{l}C_{m}(2) \cong S p(2 m, 2) \\
\cong O_{2 m+1}(2) \\
\text { on } \pm \text { hyperplanes }\end{array}$ \\
\hline LT11 & $G_{2}(2)^{\prime}$ & 28 & $\begin{array}{c}N_{M}(P) \\
P \in S y l_{3}(M)\end{array}$ & $G_{2}(2)^{\prime} \cong U_{3}(3)$ \\
\hline SP1 & $\begin{array}{c}M_{n} \\
n=11,12,22,23,24\end{array}$ & $n$ & $M_{n-1}$ & natural \\
\hline SP2 & $M^{c} L$ & 12 & $L_{2}(11)$ & \\
\hline SP3 & HS & 176 & $U_{3}(5): 2$ & \\
\hline SP4 & $\mathrm{Co}_{3}$ & 276 & $M^{c} L 2$ & \\
\hline
\end{tabular}


Hering also shows that Janko's first group $J_{1}$ cannot occur as a composition factor of $G_{0}$. His general ideas may be used to show that no sporadic simple group can occur as a composition factor. As the authors know of no published proof, we include a proof here.

PROPOSITION 3.2. With notation and hypotheses as above, $G_{0}$ has no composition factor isomo-phic to a sporadic simple group.

Proof: Assume $G_{0}$ has a sporadic simple group $D$ as a composition factor. We have already observed that $G_{0}$ may be regarded as a subgroup of $G L(n, p)$. From Dickson's Theorem [12, II, Satz 8.27] or [21, Section 3.6], we conclude $G L(2, p)$ involves no sporadic simple group. Thus, $n>2$.

Defining $\Phi_{n}^{*}(p)$ as in [9], if $\Phi_{n}^{*}(p)=1$, then by Proposition 1.4 (also [9, Theorem $3.9]), p^{n}=2^{6}$. So $G_{0} \leqslant G L(6,2)$. That is impossible because $|G L(6,2)|$ is not divisible by the order of any sporadic simple group. Thus, $\Phi_{n}^{*}(p) \neq 1$.

By hypothesis, $G_{0}$ is transitive on the nonzero vectors for $V$, so $p^{n}-1$ divides $\left|G_{0}\right|$. Since $\Phi_{n}^{*}(p) \mid\left(p^{n}-1\right), 1 \neq \Phi_{n}^{*}(p)=\left(\Phi_{n}^{*}(p),\left|G_{0}\right|\right)$. Thus, the hypotheses of Section 4 of [9] are satisfied.

Set $S=G_{0}^{(\infty)}, F=F\left(G_{0}\right)$ and $L$ the centraliser of $S$ in hom $(V, V)$. (Hering defines $S$ differently, but our definition is equivalent by $[9$, Theorem $\mathrm{B}(\mathrm{g})]$.) As in Hering, $L$ is a field of order $p^{m}$ and $V$ is a vector space over $L$ of dimension $k=n / m$. (Our $k$ is Hering's $n^{*}$.) Furthermore, elements of $G_{0}$ are semilinear transformations over $L$, so we can think of $G_{0}$ as a subgroup of $\Gamma L\left(k, p^{m}\right)$. As with $n$ above, we deduce that $k>2$.

It follows now from [9, Theorem B(d) and (a)] that $\Phi_{n}^{*}(p)=\left(\Phi_{n}^{*}(p),\left|G_{0}\right|\right)$ divides $|S F / F|$ and $S F / F \cong D$. If $F$ is not a subgroup of $C_{G_{0}}(S)$, then by [9, Theorem $\mathrm{A}(\mathrm{a})$ and $(\mathrm{c})], \Phi_{n}^{*}(p)=r=n+1=2^{a}+1$ where $r$ is a prime and $D \cong S F / F$ is faithfully represented on a vector space of dimension $2 a$ over $G F(2)$. As $r$ divides $|D|$, we examine the prime divisors of the sporadic simple groups and conclude that $r=3,5,17$ with $a=1,2,4$ respectively. However, $|D|$ doesn't divide $|G L(2 a, 2)|$ in any of the relevant cases. (Note that $a$ can be 4 only for $D \cong J_{3}, H e, F i_{23}, F i_{24}^{\prime}, B$ or $M$.) That contradiction implies $F \leqslant C_{G_{0}}(S)$. We are now in a position to bound the values for $p$ and $n$, from which the final contradiction will follow by examining a fairly large, but tractable number of cases.

First bound $n$. Let $r$ be any prime divisior of $\Phi_{n}^{*}(p)$. We've already shown that $r$ divides $|D|$. Furthermore, $n$ divides $r-1[9$, Theorem 3.5(ii)]. As an aside, we know $n>2$, so $r \geqslant 5$.

To bound $p$, we look at the structure of $G_{0}$. From $[9$, Theorem $\mathrm{B}(\mathrm{c})$ and $(\mathrm{g})], F$ is a subgroup of the multiplicative group of $L$ while $G_{0} / S F$ is isomorphic to a subgroup 
of the outer automorphism group of $D$. In particular, $\left|G_{0}\right|=|F|[S F: F]\left[G_{0}: S F\right]$ divides $\left(p^{m}-1\right)|D||O u t(D)|=\left(p^{m}-1\right)|A u t(D)|$. Recall from the transitivity of $G_{0}$ that $\left(p^{n}-1\right)$ divides $\left|G_{0}\right|$. Thus, $\left(p^{n}-1\right) /\left(p^{m}-1\right)$ divides $|A u t(D)|$. Because $\left(p^{n}-1\right) /\left(p^{m}-1\right)$ is an increasing function of $p$ for a given $n$ and $m$, we have a bound on $p$ as well as a divisibility criterion. However, for small values of $n$ and large sporadic groups, the bound is not good enough to make checking cases feasible. We need additional conditions on $n$ and $p$.

By definition, $S=S^{\prime}$ and we showed earlier that $F \leqslant C_{G}(S)$.

Thus, $S \cap F \leqslant S^{\prime} \cap Z(S)$, so $S$ is a perfect central extension of $D$ and its character table is available in the ATLAS. Now $S \leqslant G_{0} \leqslant G L(n, p)$, hence, $S$ has an $n$ dimensional representation over $G F(p)$. If $p$ does not divide $|S|$, then by a familiar result of Speiser [19, Satz 206], $n$ is the degree of an ordinary complex representation of $S$. From above, we also know that $n \leqslant r_{\max }-1$ where $r_{\max }$ is the largest prime divisor of $|D|$. We consult the character tables for the various possibilities for $S$ and find only eight cases (listed below) compatible with both conditions on $n$. In each case, $n$ is large enough that only a few primes need to be tried before $\left(p^{n}-1\right) /\left(p^{m}-1\right)$ exceeds $|A u t(D)|$.

To summarise, we have the following restrictions:

1. For every prime $r$ dividing $\Phi_{n}^{*}(p), n|(r-1), r||D|$ and $r \geqslant 5$.

2. $\left(p^{n}-1\right) /\left(p^{m}-1\right)$ divides $|A u t(D)|$ where $m=n / k<n / 2$ divides $n$.

3. Either $p$ divides $|S|$ (equivalently, $p$ divides $|D|$ ) or we have $n=10, S \cong$ $M_{11}, 2 . M_{12}, 2 . M_{22} ; n=22, S \cong M_{23} ; n=6, S \cong 2 . J_{2} ; n=18, S \cong$ $3 . J_{3}$ or $n=28, S \cong 2 . R u$.

4. $|D|$ divides $|G L(n, p)|$ (obviously).

A very modest amount of Mathematica [16] code promptly shows that the above conditions are satisfied only when $n=3, p=11$ and $D \cong J_{1} ; n=4, p=7$ and $D \cong J_{2}$ or $n=12, p=2$ and $D \cong S u z$. We restrict our attention, henceforth, to those three cases.

Since $S \leqslant G L(n, p), S$ acts on the $d=\left(p^{n}-1\right) /(p-1)$ points of the corresponding projective space. $S$ cannot stabilise a point, for if $S$ were contained in a point stabiliser, then a homomorphic image of $S$ is involved in $P G L(n-1, p)$ (see case LT1 from Proposition 3.1). Since $S$ is a perfect central extension of $D$, we conclude $D$ is involved in $L_{n-1}(p)$. That's a contradiction because in each of our three cases $|D|$ does not divide $\left|L_{n-1}(p)\right|$.

Let $S_{\alpha}$ be the point stabiliser of $\alpha$ in $S$, then $1<\left[S: S_{\alpha}\right] \leqslant d$. Let $X$ be a maximal subgroup of $S$ containing $S_{\alpha}$. It is easy to see $F \cap S=Z(S) \leqslant X$. Thus, $[S: X]=[S / F \cap S: X / F \cap S]=[F S / F: X F / F]$, which is the index in $D$ of a maximal subgroup isomorphic to $X / F \cap S$. Furthermore, $[S: X] \leqslant\left[S: S_{\alpha}\right] \leqslant d$. 
For the three cases in question, $d=133,400,4095$, respectively. Now turning to the ATLAS again, $J_{1}$ has no maximal subgroup with index less than or equal to 133 , so the first case is out. In the second case, $[S: X]$ could be 100,280 or 315 . Thus, $\left[X: S_{\alpha}\right]=\left[S: S_{\alpha}\right] /[S: X] \leqslant 400 /[S: X] \leqslant 4$ and $X$ has a homomorphic image in $S_{4}$. From the structure of $X / F \cap S$ in the ATLAS, that can only happen if $\left[X: S_{\alpha}\right]=1$. Thus, $S_{\alpha}$ is a maximal subgroup of $S$ of index 100,280 or 315 for every $\alpha$. Since the orbit lengths must add up to 400 , the only possibility is that all orbit lengths are 100. However, $J_{2}$, hence $S$, has only one class of maximal subgroups of index 100 . Therefore, $S_{\alpha}=S_{\beta}$ for some $\alpha$ and $\beta$ in different orbits. In particular, $S_{\alpha}$ equals the 2-point stabiliser $S_{\alpha \beta}$. A 2-point stabiliser in $G L(4,7)$ has a homomorphic image involved in $G L(2,7)$. (Look at the matrices or think about the stabiliser as a parabolic subgroup.) Hence, $J_{2}$ is involved in $G L(2,7)$, which is impossible. The third case is disposed of in a similar way. One argues that every point stabiliser of $S$ is a maximal subgroup of index 1782 which is impossible since 4095 is not a multiple of 1782 . That final contradiction shows that no sporadic simple group is a composition factor of $G_{\mathbf{0}}$.

The proposition below gives the possibilities for $G_{0}$ in the nonsolvable case. It and Proposition 3.1, give a classification of nonsolvable 2-transitive permutation groups. In describing the groups, one passes to the extension field $L$ of dimension $k$ over $G F(p)$ described in the proof of Proposition 3.2. Over that extension, the elements of $G_{0}$ are regarded as elements of $\Gamma L(k, q), q^{k}=p^{n}$. For ease of reference, Hering's numbering is used.

Proposition 3.3. Suppose $G$ is a nonsolvable 2-transitive permutation group having a regular normal subgroup $M$. With the notation and conventions introduced earlier, $G$ is the semidirect product of $M$ with $G_{0}$ and $G_{0}$ may be identified with one of the following:

I. $S L(n, q) \unlhd \Gamma L(k, q)$ where $q^{k}=p^{n}$, excluding the solvable cases $(k, q)=$ $(1, q),(2,2),(2,3)$.

II. $\quad S p(k, q) \unlhd \Gamma L(k, q)$ where $q^{k}=p^{n}$.

III. $\quad G_{2}\left(2^{m}\right) \unlhd G_{0} \unlhd \Gamma L(6,2)$ where $2^{6 m}=p^{n}$.

IV. $E \unlhd \Gamma L(4,3)$ where $E$ is an extra special group of order $2^{5}$ and $G_{0} / E Z\left(G_{0}\right)$ is isomorphic to a nonsolvable subgroup of $S_{5}$ [2]. (The other groups in Hering's category $I V$ are solvable.)

E1. $S L(2,5) \cong G_{0}^{(\infty)} \unlhd G_{0} \leqslant \Gamma L(2, q), q^{2}=p^{n}$ where $q=9,11,19,29,59$.

E2. $G_{0} \cong A_{6}, 2^{4}=p^{n}$.

E3. $G_{0} \cong A_{7}, 2^{4}=p^{n}$.

E4. $G_{0} \cong S L(2,13), 3^{6}=p^{n}$.

E5. $G_{0} \cong U_{3}(3), 2^{6}=p^{n}$. 
Proof: $[9,10]$ and Proposition 3.2.

We are now positioned to state and prove our main theorem.

THEOREM 3.4. $G$ is a nonsolvable group acting faithfully and 2-transitively on $S y l_{r}(G)$ if and only if $G$ and $r$ are one of the following:

a. $G \cong A_{5} \cong L_{2}(4) ; r=2$.

b. $L_{2}(5) \cong A_{5} \lesssim G \lesssim S_{5} \cong P G L(2,5) ; r=5$.

c. $\quad L_{2}(9) \cong A_{6} \lesssim G \lesssim A u t\left(A_{6}\right) \cong P \Gamma L(2,9) ; r=3$.

d. $\quad M \lesssim G \lesssim A u t(M)$ where $M \cong L_{2}\left(r^{m}\right)\left(r^{m}>3\right), U_{3}\left(r^{m}\right)\left(r^{m}>2\right)$, ${ }^{2} B_{2}\left(2^{2 m+1}\right)(m>0)$ or ${ }^{2} G_{2}\left(3^{2 m+1}\right)(m>0) ; r$ is the characteristic of the underlying field and $r$ does not divide $[G: M]$.

e. $\quad L_{2}(7) \cong L_{3}(2) \lesssim G \lesssim A u t\left(L_{3}(2)\right) \cong P G L(2,7) ; r=7$.

f. $\quad G_{2}(2)^{\prime} \cong U_{3}(3) \lesssim G \lesssim A u t\left(U_{3}(3)\right) \cong G_{2}(2) ; r=3$.

g. $G \cong{ }^{2} G_{2}(3) \cong P \Gamma L(2,8) ; r=3$ or $r=2$.

h. $G \cong M G_{0}$ where $M$ is the group of translations of a vector space of dimension 2 over $G F(q), M$ is a normal subgroup of $G, G_{0} \leqslant G L(2, q)$ with $G_{0}=G_{0}^{(\infty)} C$ where $G_{0}^{(\infty)} \cong S L(2,5), C \leqslant Z(G L(2, q)), r$ divides $|C|$ and $q=29$ or $59, r=7$ or 29 respectively.

Proof: As before, we may regard $G$ as a 2-transitive permutation group of $\operatorname{Syl}_{r}(G)$ with point stabiliser $N_{G}(P), P \in S y l_{r}(G)$. Armed with Propositions 3.1 and 3.3, we carry out the proof by checking for representations having Sylow $r$-normalisers as point stabilisers.

First suppose $M \leqslant G \leqslant \operatorname{Aut}(G)$ where $M$ is a nonabelian simple group. By Proposition 3.1, $M$ itself is 2-transitive in all cases except LT9. In that case, $G=$ ${ }^{2} G_{2}(3)$, the degree of the representation is 28 and a point stabiliser is a Borel subgroup, that is, the normaliser of a Sylow 3-subgroup. That gives part of $(g)$ with $r=3$. (The $r=2$ part will come when we consider LT2 below.) Henceforth, we may assume $M$ is 2-transitive on $\operatorname{Syl}_{r}(G)$.

For $P \in S y l_{r}(G), N_{G}(P) \leqslant N_{G}(P \cap M)$ and the 2-transitivity of $G$ implies $N_{G}(P)$ is a maximal subgroup of $G$. Therefore, either $N_{G}(P)=N_{G}(P \cap M)$ or $P \cap M \unlhd G$. The latter can happen only if $P \cap M=1$, but that's impossible because the 2-transitivity of $M$ on $S y l_{r}(G)$ implies the index in $M$ of a 2-point stabiliser is $\left[G: N_{G}(P)\right]\left(\left[G: N_{G}(P)\right]-1\right)$, which is divisible by $r$ according to the Sylow Theorems. Thus, $N_{G}(P)=N_{G}(P \cap M)$ and $N_{M}(P)=N_{M}(P \cap M)$. In particular, we seek situations where a point stabiliser in $M$ is the normaliser of a Sylow $r$-subgroup of $M$.

The structure of point stabilisers given in Proposition 3.1 rules out all remaining cases except ALT1 with $n=5$, ALT2-3, LT1- 6 and LT11 which require closer inspection. 
For ALT1 with $n=5$, the point stabiliser in $M$ is isomorphic to $A_{4}$ and is a Sylow 2-normaliser. In ALT2 and ALT3, the point stabiliser is a Sylow $r$-normaliser for $r=5$ and 3 respectively. All three cases yield examples with $G=M$. Now we need to see if there are examples with $M<G$. In ALT1, $M<G$ implies $G \cong S_{5}$ and the point stabiliser is isomorphic to $S_{4}$, not a Sylow 2-normaliser. In ALT2 and ALT3, $[\operatorname{Aut}(M): M]=2,4$ respectively, so $M$ contains all the Sylow $r$-subgroups of $G, r=3,5$ for any $G$ between $M$ and $A u t(M)$. Thus, the 2-transitivity of $M$ on $S y l_{r}(M)=S y l_{r}(G)$ guarantees the 2-transitivity of $G$. Thus, we get (a)-(c).

In case LT1, as long as $m>3$ or $q>3, P G L(m-1, q)$ has no normal Sylow subgroup, so if a point stabiliser of $M$ were a Sylow normaliser, the Sylow subgroup would have to lie in the normal subgroup of order $q^{m-1}(q-1) /(m, q-1)$. However, all the primes dividing that order also divide the order of $P G L(m-1, q)$ and so that subgroup cannot contain a Sylow subgroup of $L_{m}(q)$. When $m=3$ and $q=2$ or 3 , one may use the structure of point stabilisers from Proposition 3.1 or else the ATLAS, to deduce that the stabilisers are not Sylow normalisers. Thus, LT1 gives no examples. However, because $L_{3}(2) \cong L_{2}(7)$, we'll consider that group again in the representation of LT2.

Cases LT2-5, the Lie rank one cases, all yield possibilities with $G=M$ since point stabilisers are Borel subgroups, that is, Sylow $r$-normalisers where $r$ is the characteristic of the underlying field. It remains to see which other subgroups of $\operatorname{Aut}(M)$ are also examples, so assume $M<G \leqslant \operatorname{Aut}(M)$.

If $r$ does not divide $[G: M]$ then $S y l_{r}(G)=S y l_{r}(M)$ and $G$ inherits 2-transitivity on $S y l_{r}(G)$ from $M$. Now assume $r$ divides $[G: M]$ and $G$ is 2-transitive on $S y l_{r}(G)$. We'll show that assumption leads to a contradiction.

Since $A u t(M) / M$ is the semidirect product of a cyclic group of order $d$ with the group of field automorphisms of $G F(q)$ where $d=(2, q-1),(3, q+1), 1,1$ in cases LT2-5, respectively [20, 22, Theorem 11] and [18, Theorem 9.1], and $r$ does not divide $d$, it must be that $r$ divides the order of the group of field automorphisms. We may, therefore, assume $G$ contains a nontrivial field automorphism $\gamma$ of order a power of $r$, by replacing $G$ with a conjugate if necessary. As a consequence of the general theory of finite groups of Lie type, there is $U \in S y l_{r}(M)$ with $N_{M}(U)=U H$ where $U$ and $H$ are both invariant under the field automorphisms of $G F(q)$ and $U \cap H=1$ [4]. (Carter uses the notation $U^{1}$ and $H^{1}$ in the twisted case.) Thus, $U\langle\gamma\rangle$ is an $r$-group. Let $P$ be a Sylow $r$-subgroup of $G$ containing $U(\gamma)$. As shown earlier, $N_{M}(P)=N_{M}(P \cap M)$, but $P \cap M=U$. Therefore, $H \leqslant N_{M}(U)=N_{M}(P)$ and $[H,(\gamma)] \leqslant H \cap P=H \cap M \cap P=H \cap U=1$. That's impossible as we shall show next.

Cases LT2-4 have familiar representations as quotients of matrix groups over their 
centres where the respective natural choices for $H$ are images in the quotient of

$$
\begin{gathered}
\left\{\left(\begin{array}{cc}
k & 0 \\
0 & k^{-1}
\end{array}\right): k \in G F(q)^{\times}\right\},\left\{\left(\begin{array}{ccc}
k^{-1} & 0 & 0 \\
0 & k^{q-1} & 0 \\
0 & 0 & k
\end{array}\right): k \in G F\left(q^{2}\right)^{\times}\right\}, \\
\left\{\left(\begin{array}{cccc}
k^{l+\theta^{-1}} & 0 & 0 & 0 \\
0 & k^{\theta-1} & 0 & 0 \\
0 & 0 & k^{-\theta^{-1}} & 0 \\
0 & 0 & 0 & k^{-1-\theta^{-1}}
\end{array}\right): k \in G F(q)^{\times}\right\}
\end{gathered}
$$

where, in the latter case, $\theta$ is a certain field automorphism [12, II, 7.1 and II, 10.12] and [22, p. 133]. The field automorphism acts on the matrices by acting on the individual entries. Thus, in the unitary case LT3, for example, we have

$$
\left(\begin{array}{ccc}
\left(k^{-q}\right)^{\gamma} & 0 & 0 \\
0 & \left(k^{q-1}\right)^{\gamma} & 0 \\
0 & 0 & k^{\gamma}
\end{array}\right)=\left(\begin{array}{ccc}
k^{-q} & 0 & 0 \\
0 & k^{q-1} & 0 \\
0 & 0 & k
\end{array}\right)
$$

modulo the centre. In particular, taking $k$ to be a generator for $G F(q)^{\times}$, we deduce from the last entry that $k^{\gamma}=a k$ where $a^{3}=1$. Thus, $k^{\gamma^{3}}=a^{3} k=k$ so $\gamma$ has order 3. Moreover, $\left(k^{3}\right)^{\gamma}=a^{3} k^{3}=k^{3}$ so $\left\langle k^{3}\right\rangle$ is a subgroup of the multiplicative group of the fixed field of $\gamma$. By the Fundamental Theorem of Galois Theory, that fixed field has order $q^{1 / 3}$. Thus, $\left(q^{2}-1\right) / 3$ divides $q^{1 / 3}-1$, an impossibility. Case LT2 is handled similarly and LT4 is even easier because the centre is trivial.

For the Ree groups of LT5, a matrix representation is not very convenient so we utilise the Lie theory. Following Carter's notation [4] for generators of the group, we have $h_{a}\left(k^{\theta}\right) h_{b}(k) \in H$ for every $k \in G F\left(3^{2 m+1}\right)^{\times}$where $a$ and $b$ are fundamental roots and $\theta$ is a certain field automorphism with $3 \theta^{2}=1[4$, p.248249]. Thus, $h_{a}\left(k^{\theta}\right) h_{b}(k)=\left(h_{a}\left(k^{\theta}\right) h_{b}(k)\right)^{\gamma}=h_{a}\left(k^{\theta \gamma}\right) h_{b}\left(k^{\gamma}\right)$, or, in other words, $h_{a}\left(k^{-\theta \gamma} k^{\theta}\right)=h_{b}\left(k^{\gamma} k^{-1}\right)$. Applying these to the element $e_{a}$ of the Chevalley basis $[4$, p.92], we find $\left(k^{-\theta \gamma} k^{\theta}\right) e_{a}=\left(k^{\gamma} k^{-1}\right)^{(2(b, a)) /(b, b)} e_{a}$ and so we conclude that $\left(k^{-\theta \gamma} k^{\theta}\right)=$ $\left(k^{\gamma} k^{-1}\right)^{(2(b, a)) /(b, b)}=\left(k^{\gamma} k^{-1}\right)^{-3}[4, \mathrm{p} .45]$, or, equivalently, $\left(k^{-\theta+3}\right)^{\gamma}=k^{-\theta+3}$ for every $k \in G F\left(3^{2 m+1}\right)^{\times}$. Thus, the image of the multiplicative endomorphism $-\theta+3$ is contained in the fixed field of $\gamma$. Let $\langle k\rangle=\operatorname{ker}(-\theta+3)$, then $k^{\theta}=k^{3}$. Hence, $k^{3 \theta^{2}}=k^{9 \theta}=k^{27}$. However, $3 \theta^{2}=1$, and $|\operatorname{Im}(-\theta+e)| \geqslant\left(3^{2 m+1}-1\right) / t$. Thus, the multiplicative group of the fixed field of $\gamma$ has order at least $\left(3^{2 m+1}-1\right) / t$. By the Fundamental Theorem of Galois Theory, the fixed field of $\gamma$ has order $3^{(2 m+1) /(|(\gamma)|)} \leqslant$ $3^{(2 m+1)} / 3$. Therefore, we have the inequality $\left(3^{(2 m+1)}-1\right) / t \leqslant 3^{(2 m+1) / 3}-1$. By simple arithmetic, we conclude $t=26$ and $m=1$. In that case, $\operatorname{ker}(-\theta+3)$ is the 
entire multiplicative group of the underlying field, which means $\gamma$ fixes everything, a contradiction. The proof of (d), (e) and (g) with $r=2$ is complete.

Case LT6 and the just completed analysis shows that all possibilities are covered in (b). Similarly, LT11 yields (f).

We turn our attention to the situation where $G$ has a regular normal subgroup. In cases E2-E5 of Proposition 3.3, the point stabiliser is visibly not a Sylow $r$-normaliser for any $r$ dividing $|G|$.

For cases I, II, III and E1, suppose $G_{0}=N_{G}(P), P \in S y l_{r}(G)$. In each case, $G_{0}$ has a quasisimple normal subgroup $S$ where $S \cong S L(k, q), S p(k, q), G_{2}\left(2^{m}\right), S L(2,5)$, respectively. In no case does $S$ have a nontrivial normal Sylow subgroup. Thus, $S \cap P=$ $1, r$ does not divide $|S|$ and $P \leqslant C_{G_{0}}(S)$.

In cases I and II, $S$ itself is transitive on the nonzero vectors. It follows by a very easy argument [23, Section 4], that $P$ is semiregular and $|P|$ divides $p^{d}-1=q^{k}-1$. Since $q^{k}-1$ divides $|S|$ we contradict the results of the previous paragraph. In III, we may only get that $S$ is transitive on the $\left(q^{k}-1\right) /(q-1)$ points of the corresponding projective space $[9$, p. 444] and so we pass to homomorphic images in the group $P \Gamma L\left(6,2^{m}\right)$. An analogous argument still gives the contradiction that $r$ divides $|S|$.

In $\mathrm{E} 1$, if $r=p$, then $S \leqslant C_{G_{0}}(P) \leqslant N_{G_{0}}(P)$ which is impossible since $N_{G_{0}}(P) \leqslant$ $N_{\Gamma L(2, q)}(P)$ is a solvable group. We know from above that $r$ does not divide $|S|=$ $2^{3} \cdot 3 \cdot 5$. When $q=9,11$ or 19 , there are no primes left for $r$ and so those cases are eliminated. For $q=29$ or 59 , the only possibilities are $r=7$ or 29 , respectively. In those cases, let $a$ be an element of order $r$ in the multiplicative group of $G F(q)$. By conjugating if necessary, we may assume

$$
P \leqslant\left\{\left(\begin{array}{cc}
a^{i} & 0 \\
0 & a^{j}
\end{array}\right): 1 \leqslant i, j \leqslant r\right\}
$$

since the latter is a Sylow r-subgroup of $\Gamma L(2, q)=G L(2, q)$. If $P \neq\left\langle\left(\begin{array}{ll}a & 0 \\ 0 & a\end{array}\right)\right\rangle$, take $d=\left(\begin{array}{cc}a^{i} & 0 \\ 0 & a^{j}\end{array}\right) \in P$ with $i \neq j$. Then

$$
S \leqslant C_{G L(2, q)}(P) \leqslant C_{G L(2, q)}(d)=\left\{\left(\begin{array}{ll}
x & 0 \\
0 & y
\end{array}\right): x y \neq 0\right\}
$$

contradicting the nonsolvability of $S$. Thus, $P=\left\langle\left(\begin{array}{ll}a & 0 \\ 0 & a\end{array}\right)\right\rangle \leqslant Z(G L(2, q)) \cap G$. Taking homomorphic images in $P G L(2, q), A_{5} \cong S Z / Z \leqslant G_{0} Z / Z<P G L(2, q)$ where $Z=Z(G L(2, q))$. In $P G L(2, q), q=29,59$, all subgroups isomorphic to $A_{5}$ are maximal, so $S Z=G_{0} Z$. By the Dedekind identity, $G_{0}=S\left(Z \cap G_{0}\right)=G_{0}^{(\infty)}\left(Z \cap G_{0}\right)$. 
We have already shown $r$ divides $\left|Z \cap G_{0}\right|$. Moreover, any such subgroup of $G L(2, q)$ contains a conjugate of one of Zassenhaus's examples $[24, p .217]$ because there is only one conjugacy class of subgroups isomorphic to $S L(2,5)$ in $G L(2, q)$. Hence, any such subgroup is indeed 2-transitive on nonzero vectors. Thus, we get (h) and finish the proof of the theorem.

Two corollaries follow by inspection.

COROLLARY 3.5. Suppose $G$ is a nonsolvable finite group acting faithfully and 2-transitively on $\operatorname{Syl}_{r}(G)$ for each prime $r \in \pi$ where $\pi$ is a set of prime divisors of $|G|$ and $|\pi|>1$, then $|\pi|=2$ and either $G \cong A_{5}, \pi=\{2,5\}$ or $G \cong P \Gamma L(2,8), \pi=\{2,3\}$.

COROLLARY 3.6. Suppose $G$ is a finite group acting faithfully and 2-transitively on $S_{y l} l_{r}(G)$ for each prime $r \in \pi$ where $\pi$ is a set of prime divisors of $|G|$ and $|\pi|>2$, then $G$ is solvable.

COROLlary 3.7. Suppose $G$ is a finite group acting 2-transitively (not necessarily faithfully) on $S y l_{r}(G)$ for each prime $r$ dividing $|G|$, then $G$ is solvable.

Proof: Let $r$ be any prime dividing $|G|$. Let $R \in S y l_{r}(G)$ and recall $K_{r}=$ $\bigcap N_{G}(R)^{x}$ where the intersection is taken over all $x \in G . K_{r}$ is the kernel of the permutation representation of $G$ on $S y l_{r}(G)$, as in Section 1.

Clearly, $K_{r}$ has a normal Sylow $r$-subgroup. Thus, $\cap K_{r}$, where the intersection is taken over all primes $r$ dividing $|G|$, is nilpotent. Moreover, $G / \cap K_{r}$ embeds in $\Pi\left(G / K_{r}\right)$ where the intersection and the direct product are both taken over all primes $r$ dividing $|G|$. Consequently, to prove $G$ is solvable, it suffices to show that each $G / K_{r}$ is solvable.

$G / K_{r}$ acts faithfully and 2-transitively on $S y l_{r}(G)$ and it acts 2-transitively on $S y l_{p}(G)$ for all other primes $p$ dividing $|G|$. Assume $G / K_{r}$ is not solvable, then it is one of the groups listed in Theorem 3.4. In all but the last case, $G / K_{r}$ has no nontrivial proper normal subgroups except possibly a simple one, so $G / K_{r}$ acts faithfully and 2-transitively on all its sets of Sylow subgroups. Corollary 3.6 contradicts the nonsolvabilitiy of $G / K_{r}$. Therefore, $G / K_{r}$ has the structure given in the last case of Theorem 3.4. However, in that case, $G / K_{r}$ has a homomorphic image isomorphic to $A_{5} \cong L_{2}(5)$ and so $A_{5}$ would act 2-transitively on all of its sets of Sylow subgroups, but $A_{5}$ is not 2-transitive on its Sylow 3-subgroups. Thus, $G / K_{r}$ must be solvable as claimed.

\section{Groups $G$ 2-Transitive on $S y l_{r}(G)$ FOR EACH $r$}

From Corollary 3.7, one sees the groups mentioned in the title of this section are solvable. We remind the reader that in Section 1, we have established notation for groups similar to a subgroup of $\Gamma\left(q^{n}\right)$. 
Some additional notation is helpful here. Let $\mathcal{N}=\mathcal{N}^{1}$ denote the class of nilpotent groups and, inductively, for $i \geqslant 1, \mathcal{N}^{i+1}=\{G \mid$ There is $N \triangleleft G$ such that $N \in \mathcal{N}$ and $\left.G / N \in \mathcal{N}^{i}\right\}$. Before moving to the groups mentioned in the title of this section, we make an intermediate step.

Proposition 4.1. Suppose $G$ is 2-transitive on $S y l_{r}(G)$ for each prime $r$ and $G$ is faithful on $S y l_{p}(G)$ for some prime $p$.

(i) If $G$ on $S y l_{p}(G)$ is not similar to a subgroup of $\Gamma\left(q^{n}\right)$ on $\mathbb{K}$, then $G=$ $V \cdot S L(2,3),|V|=3^{2}$ and $p=2$.

(ii) If $G$ on $S y l_{p}(G)$ is similar to a subgroup of $\Gamma\left(q^{n}\right)$ on $\mathbb{K}$, then $G \in \mathcal{N}^{2}$ or $G \cong S_{4}, p=3$ and $q=2=n$.

Proof: By Corollary 3.7, $G$ is solvable and we apply the results from Section 2.

(i) By inspecting the groups listed in Proposition 2.3, one confirms that the only group satisfying our stronger condition is (i), where $|V|=3^{2}$ and $G=V \cdot S L(2,3)$, with $p=2$.

(ii) Now suppose $G$ on $S y l_{p}(G)$ is similar to the subgroup $\bar{G} \leqslant \Gamma\left(q^{n}\right)$, but $G \notin$ $\mathcal{N}^{2}$. Let $\bar{P} \in S y l_{p}(\bar{G})$.

From the 2-transitivity of $\bar{G}$ on $\mathbb{K},|\bar{G}|=q^{n}\left(q^{n}-1\right) m$ where $m \mid n$. Moreover, using Proposition 1.2, it is straightforward to deduce that $V \leqslant \bar{G}$ and $V$ is the unique minimal normal subgroup of $\bar{G}$. Also $C_{\bar{G}}(V)=V$.

STEP 1. For all primes $r \neq q$ such that $r|| G \mid, G$ on $S y l_{r}(G)$ is similar to $G$ on $S y l_{p}(G)$.

Let $r$ be any prime divisor of $|\bar{G}|, r \neq q$. If $\bar{K}_{r} \neq 1$, then $V \leqslant \bar{K}_{r} \leqslant N_{\bar{G}}(\bar{R})$. Thus $[R, V]=1$ and so $\bar{R} \leqslant C_{\bar{G}}(V)=V$. Hence $\bar{K}_{r}=1$ for any prime divisor of $|\bar{G}|, r \neq q$. Then $G$ is 2-transitive and faithful on $S y l_{r}(G)$. Moreover, by Proposition $1.2, N_{\bar{G}}(R)$ is a complement to $V$ in $\bar{G}$. This leads to an application of Ore's Theorem and the statement in Step 1, as in [12, II, Satz 3.8].

STEP 2. $m$ is a power of $q$ and $V S \leqslant \bar{G}$.

Since $G \notin \mathcal{N}^{2}$, if $q$ is Mersenne, $n \neq 2$ by Proposition 2.2. Thus by Proposition 2.1, if $r$ is a prime, $r|| G \mid, r \neq q$, and $\bar{R} \in S y l_{r}(G)$, from Step 1 it follows that $\bar{R} \leqslant V S$. Yet $|G|=q^{n}\left(q^{n}-1\right) m=|V S| m$. Thus $m$ is a power of $q$.

Moreover $V \leqslant \bar{G}$ and for each prime $r|| G \mid, r \neq q$ and $\bar{R} \in S y l_{r}(G), \bar{R} \leqslant V S$. So $V S \leqslant \bar{G}$. This gives Step 2 and, then, the next step is transparent.

STEP 3. $\bar{G}=V S\langle\tau\rangle$ where $\langle\tau\rangle \leqslant\langle\sigma\rangle$ and $|\langle\tau\rangle|=m$.

STEP 4. There is a Zsigmondy prime $r$ for the pair $q$ and $n$.

Suppose not. By Proposition 1.5 and the argument in Step 2, it must be that $q=2$ and $n=6$. 
Then $|G|=2^{6} \cdot(9 \cdot 7) \cdot 2$ from Step 2. It is easy to verify that the subgroup $H \leqslant \Gamma\left(2^{6}\right)$ with $|H|=2^{6}(9 \cdot 7) \cdot 2$ and $V S \leqslant H$, is not 2-transitive on $S y l_{2}(H)$. This verifies Step 4.

STEP 5. $G / K_{q}$ does not have a normal Sylow $q$-subgroup and $G / K_{q}$ is a supersolvable group which acts faithfully and 2-transitively on $S y l_{q}\left(G / K_{q}\right)$. Consequently $\left|G / K_{q}\right|=$ $s(s-1)$ where $s$ is a prime and $s-1$ is a power of $q$.

If $Q \in S y l_{q}(G)$ and $K_{q} Q / K_{q} \triangleleft G / K_{q}$, then $K_{q} Q \leqslant N_{G}(Q)$ and $K_{q} Q \triangleleft G$. Hence $Q \triangleleft G$. From Step 2 and Step 3 , and the fact that $S$ is cyclic, $G / V$ has a normal Sylow subgroup for each prime $t, t \neq q$. Consequently $G / V$ is nilpotent, contrary to the hypothesis that $G \notin \mathcal{N}^{2}$. So $G / K_{q}$ does not have a normal Sylow $q$-subgroup.

That $G / K_{q}$ is supersolvable follows since $V \leqslant \bar{K}_{q}$ and $\Gamma\left(q^{n}\right) / V \cong S\langle\sigma\rangle$ is supersolvable. By hypothesis, $G$ is 2-transitive on $S y l_{q}(G)$ and so $G / K_{q}$ is faithful and 2transitive on $S y l_{q}\left(G / K_{q}\right)$. Since $G / K_{q}$ does not have a normal Sylow $q$-subgroup, it follows, from Proposition 1.2, that $\left|G / K_{q}\right|=s(s-1)$, for a prime $s$, and $q \mid s-1$. Because $\bar{G} / V$ has normal Sylow $t$-subgroups for $t \neq q$ and $V \leqslant \bar{K}_{q}, s-1$ is a power of $q$.

STEP 6. $V \in S y l_{q}\left(\bar{K}_{q}\right)$ and so $m=s-1$.

Let $X \in S y l_{q}\left(\bar{K}_{q}\right)$. Note $\bar{K}_{q}$ has a normal Sylow $q$-subgroup. Also $V \leqslant X$ and so $V \cap Z(X) \neq 1$. $V \cap Z(X) \triangleleft \bar{G}, V$ is the minimal normal subgroup of $\bar{G}$ and so $V \leqslant Z(X)$. Hence $X \leqslant C_{G}(V)=V$. Thus $V=X$.

Thus the order of a Sylow $q$-subgroup of $\bar{G} / V$ is the same as the order of a Sylow $q$-subgroup of $\bar{G} / \bar{K}_{q}$. That is, $m=s-1$.

Since $m>1$, we obtain

STEP 7. $s$ is odd and $q=2$.

STEP 8. $s=r$.

The prime $s$ is the only odd prime for which a Sylow $s$-subgroup of $G$ is not contained in $K_{2}$. Note that $V\langle\tau\rangle \in S y l_{2}(\bar{G})$ and if, for $\bar{R} \in S y l_{r}(G)$ with $\bar{R} \leqslant$ $S, \bar{R} \leqslant \bar{K}_{2}$, then $[\bar{R},\langle\tau\rangle] \leqslant \bar{R} \cap V\langle\tau\rangle=1$. This would mean that $\bar{R}$ is contained in the fixed field of $\langle\tau\rangle$, contrary to the fact that $r$ is a Zsigmondy prime for $2, n$. Thus $s=r$.

STEP 9. If $\mathbb{L}$ is subfield of $\mathbb{K}$ fixed by $\langle\tau\rangle$, then $\mathbb{L}$ is a maximal subfield of $\mathbb{K}$.

From Galois theory, $|\mathbb{K}: \mathbb{L}|=m$ is a power of 2. Also $r$ is a Zsigmondy prime for 2 and $n$. Suppose $\mathbb{L}<\mathbb{L}_{0}<\mathbb{K}$ with $\left|\mathbb{L}_{0}\right|=2^{n_{0}}$. If $n_{0} \neq 6$, choose $t$ a Zsigmondy prime for 2 and $n_{0}$, or if $n_{0}=6$, choose $t=3$. In either case, $t \neq r$. Note that when $n_{0}=6, \mathbb{L}=G F\left(2^{3}\right)$ since $m$ is a power of 2 . Following the usual correspondence into $\bar{G}$, if $\bar{T} \in S y l_{t}(\bar{G})$ with $\bar{T} \leqslant S, \bar{T}$ is not contained in the fixed field of $\langle\tau\rangle$, but, 
because $t \neq r, \bar{T}$ must be contained in the fixed field of $\langle\tau\rangle$ by the argument in Step 8 . Consequently $\mathbb{L}$ is a maximal subfield of $\mathbb{K}$.

STEP 10. The conclusion.

The degree of $\mathbb{K}$ over a maximal subfield is a prime and so $m=2$. But then from Step 6, $s=3$ and from Step 8 and Step 4, 3 is a Zsigmondy prime for 2, $n$. It follows that $n=2$ and $G \cong S_{4}$.

In Corollary 3.7 the subgroup $\bigcap K_{r}$ played a role. More can be said. One can $r|| G \mid$

show that $\bigcap_{r|| G \mid} K_{r}=Z_{\infty}(G)$, the hypercenter of $G$. We omit the proof since in the cases where we use it, $G$ is solvable and this fact is included in standard references [12, VI, Satz $11.11 \mathrm{c}]$ and [8, Theorem 5.a,b].

Note if $H \leqslant G$ such that $Z_{\infty}(G) \leqslant H, Z_{\infty}(G) \leqslant Z_{\infty}(H)$, and if $G / Z_{\infty}(G) \in \mathcal{N}$, then $G=Z_{\infty}(G) \in \mathcal{N}$. It follows that for any $i$, if $G / Z_{\infty}(G) \in \mathcal{N}^{i}$, then $G \in \mathcal{N}^{i}$.

Proposition 4.2. Suppose $G$ is 2-transitive on $S y l_{r}(G)$ for each prime $r$. Then $G \in \mathcal{N}^{3}$. Further, if $\left|S y l_{3}(G)\right|=1$ or $\left|S y l_{2}(G)\right|=1, G \in \mathcal{N}^{2}$ and if for $T \in S y l_{2}(G), T \leqslant Z_{\infty}(G)$, then $G \in \mathcal{N}$.

ProOF: We have seen $G / Z_{\infty(G)}$ is isomorphic to a subgroup of $\prod G / K_{p}$. $p|| G \mid$

For each $p, G / K_{p}$ is 2-transitive on $S y l_{r}\left(G / K_{p}\right)$ for each prime $r$ and faithful on $S y l_{p}\left(G / K_{p}\right)$ and so from Proposition $4.2, G / K_{p} \in \mathcal{N}^{3}$. Consequently $G / Z_{\infty}(G) \in \mathcal{N}^{3}$ and thus $G \in \mathcal{N}^{3}$.

Note that for any prime $r$, if $\left|S y l_{r}(G)\right|=1$, then for any $N \triangleleft G,\left|S y l_{r}(G / N)\right|=1$. Inspection of the two possibilities for $G / K_{p}$ which are not in $\mathcal{N}^{2}$ shows neither has a normal Sylow 2-subgroup nor a normal Sylow 3-subgroup. Consequently $G / Z_{\infty}(G) \in$ $\mathcal{N}^{2}$ and so $G \in \mathcal{N}^{2}$.

Finally, a faithful non-identity 2-transitive group must have even order and so $G=K_{p}$ for each $p$ and thus $G \in \mathcal{N}$.

Frankly, we had hoped for a complete classification of groups $G$ which are 2transitive on $S y l_{r}(G)$ for each prime $r$. Let $\mathcal{X}=\left\{G \mid G\right.$ is 2-transitive on $S y l_{r}(G)$ for each prime $r$. Certainly $\mathcal{X}$ is quotient-closed, but direct products are more subtle.

Observe that, in general, if $G_{1}$ is 2-transitive on $\Gamma_{1}$ and $G_{2}$ is 2-transitive on $\Gamma_{2}$, then $G_{1} \times G_{2}$ acting componentwise on $\Gamma_{1} \times \Gamma_{2}$ is 2-transitive if and only if $\left|\Gamma_{1}\right|=1$ or $\left|\Gamma_{2}\right|=1$.

Corollary 4.3. Suppose $\left\{G_{1}, G_{2}\right\} \subseteq \mathcal{X}$. Then $G_{1} \times G_{2} \in \mathcal{X}$ if and only if $\left|S y l_{r}\left(G_{2}\right)\right|=1$ for each prime $r$ such that $\left|S y l_{r}\left(G_{1}\right)\right| \neq 1$.

Of deeper concern is classifying those groups $G$ such that $G / Z_{\infty}(G)$ is one of 
our known $\mathcal{X}$-groups, and whether $G \in \mathcal{X}$ implies characterisable restrictions on $K_{p} / Z_{\infty}(G)$ for some prime $p$.

\section{REFERENCES}

[1] S.S. Abhyankar, 'Galois theory on the line in nonzero characteristic', Bull. Amer. Math. Soc. (N.S.) 27 (1992), 68-133.

[2] J. André, 'Projektive Ebenen über Fastkörpern', Math. Z. 62 (1955), 137-160.

[3] B. Brewster and M. Ward, 'Finite solvable groups acting 2-transitively on Sylow subgroups', in Group theory, Proceedings of the Biennial Ohio State-Denison Conference, May 1992 (World Scientific, Singapore, New Jersey, London, Hong Kong), pp. 322-325.

[4] R.W. Carter, Finite groups of Lie type (John Wiley \& Sons, London, New York, Sydney, Toronto, 1972).

[5] P.J. Cameron, 'Finite permutation groups and finite simple groups', Bull. London Math. Soc. 13 (1981), 1-22.

[6] J.H. Conway, R.T. Curtis, S.P. Norton, R.A. Parker and R.A. Wilson, Atlas of finite groups (Clarendon, Oxford, 1985).

[7] C.W. Curtis, W.M. Kantor and G.M. Seitz, 'The 2-transitive permutation representations of the finite Chevalley groups', Trans. Amer. Math. Soc. 218 (1976), 1-59.

[8] K. Doerk and T. Hawkes, Finite soluble groups (Walter de Gruyter, Berlin, New York, 1992).

[9] C. Hering, 'Transitive linear groups and linear groups which contain irreducible subgroups of prime order', Geom. Dedicata 2 (1974), 425-460.

[10] C. Hering, 'Transitive linear groups and linear groups which contain irreducible subgroups of prime order, II', J. Algebra 93 (1985), 151-164.

[11] B. Huppert, ' $Z$ weifach transitive, aufösbare Permutationsgruppen', Math. Z. 68 (1957), 126-150.

[12] B. Huppert, Endliche Gruppen I (Springer-Verlag, Berlin, Heidelberg, New York, 1967).

[13] B. Huppert and N. Blackburn, Finite groups II (Springer-Verlag, Berlin, Heidelberg, New York, 1982).

[14] W.M. Kantor, 'Some consequences of the classification of finite simple groups', in Finite groups-Coming of age, Proceedings of the Canadian Mathematical Society Conference held on June 15-28, 1982 (American Mathematical Society, Providence, 1985), pp. 159-173.

[15] E. Maillet, 'Sur les isomorphes holoédriques et transitifs des groupes symétriques ou alternés', J. Math. Pures Appl. 1 (1895), 5-34.

[16] Mathematica: A System for Doing Mathematics by Computer (Wolfran Research, Inc., Champaign, 1993).

[17] A. Playtis, S. Sehgal and H. Zassenhaus, 'Equidistributed permutation groups', Communications in Algebra 6 (1978), 35-57.

[18] R. Ree, 'A family of simple groups associated with the simple Lie algebra $G_{2}$ ', Amer. J. Math. 83 (1961), 432-462.

[19] A. Speiser, Die Theorie der Gruppen von endlicher Ordnung, 3. Auf. (Springer, Berlin, 
1937 (U.S. Publication: Dover, New York, 1945)).

[20] R. Steinberg, 'Automorphisms of finite linear groups', Canad. J. Math. 9 (1959), 875-891.

[21] M. Suzuki, Group Theory I (Springer-Verlag, Berlin, Heidelberg, New York, 1982).

[22] M. Suzuki, 'On a class of doubly transitive groups', Ann. Math. 75 (1962), 105-145.

[23] H. Wielandt, Finite permutation groups (Academic Press, New York and London, 1964).

[24] H. Zassenhaus, 'Über endliche Fastkörper', Abh. Math. Sem. Univ. Hamburg 11 (1936), 187-220.

Department of Mathematical Sciences

SUNY - Ringhamton

Binghamton NY 13901

United States of America
Department of Mathematics

Bucknell University

Lewisburg PA 17839

United States of America

e-mail: mward@bucknell.edu 